\title{
Die Zusammenarbeit mit der IV wird immer problematischer
}

\author{
Die Zusammenarbeit der IV-Organe mit uns Grundversorgern hat ein absolut \\ erschreckendes Niveau angenommen. Zu einer zunehmenden Bürokratisierung \\ gesellt sich ein Umgangston, der mich ärgert. Die folgenden Kritikpunkte wurden \\ der Direktion der SVA Zürich vom Autor Anfang Oktober zur Kenntnis gebracht. Bis \\ zum Zeitpunkt der Drucklegung dieses Beitrags ist eine Antwort ausgeblieben.
}

Felix Tapernoux

Korrespondenz: Dr. med. Felix Tapernoux Facharzt für Allgemeinmedizin FMH Ferrachstr. 29 CH-8630 Rüti
Seit der 5. IV-Revision, einem Geschenk des Parlaments an die Taggeldversicherungen und erst in zweiter Linie einer Möglichkeit der Frühintegration, müssen wir auf Druck der Taggeldversicherungen immer mehr IV-Anträge ausfüllen, wobei 8 von 10 sinnlos sind, weil noch völlig unklar ist, ob der Patient wieder voll arbeitsfähig wird. Gemäss Aussagen des hiesigen Handchirurgen sind es bei seinen Patienten fast alle, da die Heilung einfach länger dauert ...

\section{Unsinnige Praktiken}

Es ist unsinnig, dass (Taggeld-)Versicherungen die versicherten Arbeitnehmer in reinem Eigeninteresse, ohne Rücksprache mit den behandelnden Ärzten, dazu auffordern dürfen, eine IV-Anmeldung zu machen. Kürzlich hat das Parlament beschlossen, dass die Versicherungen selber IV-Anmeldungen machen können, was uns sicherlich noch mehr unsinnige IV-Zeugnisse bescheren wird. In einer entsprechenden Aufforderung an eine Patientin schreibt die CSS wörtlich: «Allenfalls zuviel bezahlte Taggelder können wir dann direkt bei der IV-Stelle zurückfordern, und die Zusammenarbeit mit der IV-Stelle wird vereinfacht.» Darum geht es, und um nichts anderes.

Dass dieselbe Versicherung noch die Frechheit hatte, dem Arbeitgeber der Patientin eine Kopie dieser Anmeldungsaufforderung zuzustellen, ist im Sinn der von der IV angestrebten Reintegration absolut kontraproduktiv. Ein solches Vorgehen könnte bei einigen Arbeitgebern erst recht zu einer Kündigung führen, wenn diese sehen, dass eine dauernde Invalidität droht. Dabei handelte es sich im konkreten Fall lediglich um einen Hüftgelenksersatz, der leider mit einer Frühlockerung einherging.

Ebenso unverständlich ist es, dass Sozialämter via IV oder direkt (?) eine Begutachtung bei einer der ohnehin überlasteten Begutachtungsstellen bewirken können, selbstverständlich ebenfalls hinter unserem
Rücken! In dieses Bild passt der Vorschlag, dass künftig die funktionelle Leistungsfähigkeit der versicherten Person ausschliesslich durch die regionalen ärztlichen Dienste (RAD) beurteilt werden soll [1].

$\mathrm{Ob}$ frühe IV-Anmeldungen tatsächlich in einigen Fällen zu Frühintegration bzw. dem Erhalt des bisherigen Arbeitsplatzes dank Intervention der IV geführt haben, kann ich natürlich nicht beurteilen. Allerdings habe ich dies bisher bei keinem einzigen Patienten erlebt. Es ist vielmehr so, dass die IV bei diesen laufenden «Fällen» gezwungen ist, dauernd Folgezeugnisse zu verlangen.

\section{Rentenrevisionen ohne Nutzen}

$\mathrm{Zu}$ einer weiteren Häufung an IV-Zeugnissen führen vermehrte Rentenrevisionen, die wir wohl der Scheininvalidendiskussion der SVP zu verdanken haben. «Die neuerdings geforderte Überprüfung aller alten Renten ist den Preis nicht wert, den sie kostet», schrieb der langjährige MEDAS-Chefarzt Dr. C. Schuler [2].

Mehr noch als die Flut an administrativer Büroarbeit ärgert mich die Art und Weise, wie Funktionäre der Krankenkassen und Versicherungen mit uns umgehen, wobei die IV offensichtlich eine Vorreiterrolle einnimmt.

Früher war es eine Selbstverständlichkeit, uns ein spezielles Revisionsformular mit dem bisherigen IV-Grad zuzustellen. Nun hat man dieses abgeschafft, vermutlich, damit die Mitarbeiter dieselben Autotextbausteine verwenden können. Ich schicke jedes Revisionszeugnis zurück mit der Bitte, mir den bisherigen IV-Grad mitzuteilen, als minimales Feedback für meine früheren Bemühungen. Bisher wurde meinem Wunsch umgehend entsprochen. Kürzlich bekam ich jedoch folgende Antwort: «Leider können wir Ihrem Gesuch nicht entsprechen, da der behördliche IV-Grad für die medizinische Berichterstattung nicht relevant ist.» Mein zweiter Brief an die Direktion der SVA mit der Feststellung, ich könnte ebenso gut argumentie- 
ren, «die medizinische Berichterstattung ist für die behördliche Festlegung des IV-Grades irrelevant», ein Eindruck, der sich ohnehin inzwischen festgesetzt hat, ist auch noch nicht beantwortet worden.

Seit Februar 2010 werden wir nicht nur gemahnt, wenn ein IV-Zeugnis nach 30 Tagen nicht eingetroffen ist. Auch die Patienten erhalten eine Kopie der Mahnung, um Druck zu machen und uns gegenüber dem Patienten für die Verzögerung verantwortlich zu

\section{Zu einer zunehmenden Büro- kratisierung gesellt sich ein Umgangston, der mich ärgert.}

machen. Skrupellos werden wir von der IV als Hauptverantwortliche für die lange Verfahrensdauer gegenüber den Patienten und auch in den Medien [3] dargestellt: «Von dieser (IV) hat sie in den vergangenen Monaten aber auf Anfrage immer dieselbe Antwort erhalten: Man müsse erst die Arztberichte abwarten» ist in diesem Artikel zu lesen. Da die Patienten oftmals mit dem IV-(Vor-)Bescheid zu uns kommen, ist uns der «Delay» bei der IV durchaus bekannt. Wenn ich die IV um eine Auskunft bitte, dauert es zwei Monate, um in zwei Sätzen zu erfahren, dass sie nicht zuständig seien.

\section{Staatsverdrossenheit nimmt zu}

Oftmals wundert man sich, dass Ärzte bei der IV angestellt sind. So wurde kürzlich in der TV-Sendung Kassensturz der absolut klare «Fall» einer Patientin mit Morbus Behçet vorgestellt, die erst nach Abklärungen bei der MEDAS und dank Medienpräsenz nach über 2 Jahren zu ihrer Rente kam. Ich habe auch eigene Fälle, bei denen ich mich über die geringe Entscheidungsfreudigkeit der IV-Ärzte gewundert habe.
Der ehemalige AGZ-Präsident Dr. W. Grete hat in einem äusserst bemerkenswerten Artikel [4] eindrücklich vor den Folgen der Staatsverdrossenheit von uns Ärzten an der Basis gewarnt. Tatsächlich hat sich das Verhältnis zwischen den Versicherungen und uns Ärzten in den letzten Jahren zunehmend verschlechtert. Wir kommen uns immer mehr als nützliche Idioten vor, die gefälligst möglichst rasch immer mehr (unpassende) Autotextbaustein-Fragen zu beantworten haben, von Zusammenarbeit kann keine Rede mehr sein. Es ist wirklich allerhöchste Zeit, dass die Versicherungen und speziell die IV eine Änderung ihrer Politik umsetzen.

Dr. W. Grete hat im erwähnten Artikel den Weg vorgezeichnet: «Ärztinnen und Ärzte sind durch die Versicherungsmediziner grundsätzlich über abweichende Entscheidungen zu informieren. Nur so entsteht ein allfälliger Lerneffekt. Das Versteckspiel hinter dem Datenschutz ist dabei zu hinterfragen. Bestehende Fronten zwischen Vertrauensärzten und frei praktizierenden Kollegen müssen abgebaut werden. Dazu gehört auch ein von gegenseitiger Achtung getragener Umgangston.»

In einer der nächsten SÄZ-Ausgaben ist eine Stellungnahme von Stefan Ritler, Leiter des Geschäftsfelds Invalidenversicherung des Bundesamtes für Sozialversicherung (BSV), vorgesehen.

\section{Literatur}

1 Romann C, Kuhn HP. IV: Wiedereingliederung braucht echte Anreize für Arbeitgeber. Schweiz Ärztezeitung. 2010;91(46):1803.

2 Schuler C. Gesund unpässlich, unwohl, krank invalid: Spiel ohne Grenzen. Schweiz Ärztezeitung. 2010;91(34):1299-1302.

3 Fischer A. Das lange Warten auf Wiedereingliederung. Tages-Anzeiger vom 27.9.2010.

4 Grete W. Die Staatsverdrossenheit der Ärzteschaft ein Risikofaktor für den Sozialstaat. Schweiz Ärztezeitung. 2010;91(37):1439-41. Grete W. Die Solidarität der Ärzte mit dem Sozialstaat bröckelt. NZZ am Sonntag vom 17.10.2010. 